\title{
Design of the South East Asian Nutrition Survey (SEANUTS): a four-country multistage cluster design study
}

\author{
Anne Schaafsma ${ }^{1 *}$, Paul Deurenberg ${ }^{2}$, Wim Calame ${ }^{3}$, Ellen G. H. M. van den Heuvel ${ }^{1}$, \\ Christien van Beusekom ${ }^{1}$, Jo Hautvast ${ }^{4}$, Sandjaja ${ }^{5}$, Poh Bee Koon ${ }^{6}$, Nipa Rojroongwasinkul ${ }^{7}$, \\ Bao Khanh Le Nguyen ${ }^{8}$, Panam Parikh ${ }^{1}$ and Ilse Khouw ${ }^{1}$ on behalf of the SEANUTS Study Group \\ ${ }^{1}$ FrieslandCampina, Amersfoort, The Netherlands \\ ${ }^{2}$ Nutrition Consultant, Telega Harbour Marina, Lot 1, Langkawi 07000, Malaysia \\ ${ }^{3}$ StatistiCal BV, Wassenaar, The Netherlands \\ ${ }^{4}$ Jo Hautvast, Hamelakkerlaan 30, 6703 EK Wageningen, The Netherlands \\ ${ }^{5}$ Pesatuan Abli Gizi Indonesia (PERSAGI), Bogor 16112, Indonesia \\ ${ }^{6}$ Universiti Kebangsaan Malaysia (UKM), 50300 Kuala Lumpur, Malaysia \\ ${ }^{7}$ Institute of Nutrition, Mabidol University, Nakhon Pathom 73170, Thailand \\ ${ }^{8}$ National Institute of Nutrition (NIN), Hanoi, Vietnam
}

(Submitted 19 September 2012 - Final revision received 10 December 2012 - Accepted 14 January 2013)

\begin{abstract}
Nutrition is a well-known factor in the growth, health and development of children. It is also acknowledged that worldwide many people have dietary imbalances resulting in over- or undernutrition. In 2009, the multinational food company FrieslandCampina initiated the South East Asian Nutrition Survey (SEANUTS), a combination of surveys carried out in Indonesia, Malaysia, Thailand and Vietnam, to get a better insight into these imbalances. The present study describes the general study design and methodology, as well as some problems and pitfalls encountered. In each of these countries, participants in the age range of $0.5-12$ years were recruited according to a multistage cluster randomised or stratified random sampling methodology. Field teams took care of recruitment and data collection. For the health status of children, growth and body composition, physical activity, bone density, and development and cognition were measured. For nutrition, food intake and food habits were assessed by questionnaires, whereas in subpopulations blood and urine samples were collected to measure the biochemical status parameters of Fe, vitamins A and D, and DHA. In Thailand, the researchers additionally studied the lipid profile in blood, whereas in Indonesia iodine excretion in urine was analysed. Biochemical data were analysed in certified laboratories. Study protocols and methodology were aligned where practically possible. In December 2011, data collection was finalised. In total, 16744 children participated in the present study. Information that will be very relevant for formulating nutritional health policies, as well as for designing innovative food and nutrition research and development programmes, has become available.
\end{abstract}

Key words: Nutrition survey: South East Asia: Infants: Children: Design

Many South East Asian countries are in transition due to rapid industrialisation, with concurrent changes in dietary habits and lifestyle patterns in all age groups. Such changes have also taken place in other parts of the world and are known to increase the risk of chronic diseases later in life, as well as to limit development and cognitive performance early in life. Over the past 200 years, major changes have taken place in the Western diets such as an increase in refined sucrose consumption from $5 \mathrm{~kg}$ per capita per year in 1815 up to about $70 \mathrm{~kg}$ in 2000 , changes in ratios between as well as in absolute amounts of macronutrients, lower intakes of micronutrients and dietary fibres, a change from alkaline to acidic metabolism, and generally a higher Na consumption ${ }^{(1)}$. It is striking to note that worldwide 2 billion people have a micronutrient deficiency, which may interfere with adequate cognitive development and performance in children ${ }^{(2,3)}$. This estimate does not even include people with the so-called hidden deficiencies due to status parameter cut-off levels that are not in pace with the latest scientific insights, such as those for vitamin $\mathrm{D}^{(4)}$.

Abbreviations: DXA, dual-energy X-ray absorptiometry; SEANUTS, South East Asian Nutrition Survey. 
To identify the changes in diet and dietary habits and their consequences for health and development, surveys such as the National Health and Nutrition Examination Survey (NHANES) in USA ${ }^{(5)}$ (started in 1960), the Healthy Lifestyle by Nutrition in Adolescence (HELENA) in Europe ${ }^{(6)}$ (started in 2002) and the Feeding Infants and Toddlers Study (FITS) 2008 in $\mathrm{USA}^{(7)}$ have been conducted.

Although many countries in South East Asia had conducted surveys in the past, it was believed that health and nutrition information, especially in older infants and children, was lacking or no longer up to date. With this background, FrieslandCampina (Amersfoort, The Netherlands) decided to initiate and facilitate the South East Asian Nutrition Survey (SEANUTS) in collaboration with four leading research institutes in Indonesia, Malaysia, Thailand and Vietnam. The project aimed to provide insights into dietary intakes, food habits, nutritional status, growth, body composition, physical activity, and cognitive development and performance in a country-representative sample of $0.5-$ to 12 -year-old infants and children. The present study describes the general study design and methodology and discusses some problems and pitfalls encountered along the way.

\section{Study design}

Study protocols were prepared by all parties involved: FrieslandCampina; J. Hautvast (Emeritus professor of Human Nutrition, University Wageningen, The Netherlands); Dr P. Deurenberg (nutrition consultant, Malaysia); the renowned and experienced research partners mentioned in Table 1, who also took care of the executions of protocols. The final study protocols were presented during a meeting with all experts involved in Kuala Lumpur on 4 March 2010. The primary goal of the project was to collect detailed information on dietary intake, food habits, physical activity, bone density, development and cognition, growth and body composition per country. The secondary goal was to combine the outcomes of the four countries to be able to prepare a regional statement with regard to the measured parameters.

Recruitment of a representative group of boys and girls from both urban and rural areas was population based or based on schools, kindergartens and nurseries, using a cross-sectional study design based on multistage cluster/stratified random sampling (see the Statistics section). In total, 16744 apparently healthy children were recruited (Tables 2 and 3). The participants did not exhibit any sign of physical, clinically mental or clinically genetic disorders and were capable of participating in the study. Parents and/or carers approved participation by providing written consent. Exclusion criterion for participation was age $<6$ months and $>12$ years (except for Vietnam, which used 11 years as a cut-off, which is the upper age for primary school). Measurements were carried out at or near recruitment locations (schools or health centres) except for the dual-energy X-ray absorptiometry (DXA) measurement in a selected urban population, which was done in hospitals. The methodology was standardised when feasible allowing comparison of results between the countries, as a secondary aim. Results will be published in peer-reviewed international journals. Detailed results from subpopulations will also be used for educational purposes such as in the preparation of thesis manuscripts. The study was conducted according to the guidelines laid down in the Declaration of Helsinki. All procedures involving the participants were approved by the Medical Ethical Committees of the participating institutes. The present study is registered in the Netherlands Trial Registry as NTR2462.

In each of the countries, dietary intake and food habits were studied using age-specific and validated questionnaires, whereas in subpopulations blood $(5-10 \mathrm{ml}$ venous blood for the older children and capillary (finger prick) blood for the younger children) and urine samples were collected to study the biochemical status parameters of Fe, vitamins A and D, DHA, and lipid profile (the latter in only Thailand) and urinary iodine (only Indonesia). Inflammation markers (serum highsensitivity C-reactive protein and $\alpha-1$ glycoprotein) were analysed in blood samples since chronic inflammation and infection are potential confounders of serum ferritin and retinol. Using both acute-phase proteins (high-sensitivity C-reactive protein $>5 \mathrm{mg} / \mathrm{l}$ and/or $\alpha-1$ glycoprotein $>1 \mathrm{~g} / \mathrm{l}$ ), children can be classified into incubation, early-convalescence or late-convalescence groups ${ }^{(8)}$. Blood and urine samples were analysed in accredited central commercial clinical chemical laboratories and by the research institutes themselves, depending on the methodology needed. Physical activity was studied using age-specific questionnaires and in randomly selected subpopulations by pedometer counts. Bone quality and bone mineral density were measured with a mobile qualitative ultrasound device and DXA, respectively. The latter method was used only in the urban subpopulations of Jakarta (nearby Yogyakarta), Kuala Lumpur, Bangkok and Hanoi due to the availability of the equipment in hospitals. Development and cognition were studied using questionnaires and specific tests that could be easily applied in the field. For the anthropometric measurements, digital weighing scales (weight), stadiometers (height and sitting height) and calipers (skinfolds and skeletal widths) were used. Circumferences of mid-upper arm, waist and head (in only Malaysia for participants aged

Table 1. Research partners participating in the South East Asian Nutrition Survey (SEANUTS)

\begin{tabular}{lll}
\hline Country & Institute & Principal investigator \\
\hline Indonesia, Bogor & PERSAGI (Indonesian Nutrition Association) & Dr Sandjaja \\
Malaysia, Kuala Lumpur & Universiti Kebangsaan Malaysia & Dr Poh Bee Koon \\
Thailand, Nakhon Pathom & Mahidol University Thailand & Dr Nipa Rojroongwasinkul \\
Vietnam, Hanoi & Vién Dinh Duõng (National Institute of Nutrition) & Dr Le Nguyen Bao Khanh \\
\hline
\end{tabular}


Table 2. Overview of the number of participants in each country by age category ${ }^{\star}$

\begin{tabular}{|c|c|c|c|c|c|c|c|}
\hline \multicolumn{2}{|c|}{ Indonesia } & \multicolumn{2}{|c|}{ Malaysia } & \multicolumn{2}{|c|}{ Thailand } & \multicolumn{2}{|c|}{ Vietnam } \\
\hline Age (years) & $n$ & Age (years) & $n$ & Age (years) & $n$ & Age (years) & $n$ \\
\hline $0.5-1$ & 2392 & $0.5-0.9$ & 68 & $0.5-0.9$ & 142 & $0.5-5.9$ & 952 \\
\hline $2-5$ & 2413 & $1-3$ & 538 & $1-2$ & 547 & $6-11.9$ & 1920 \\
\hline \multirow[t]{2}{*}{$6-12$} & 2406 & $4-6$ & 967 & $3-5$ & 860 & & \\
\hline & & $7-12$ & 1969 & $6-11$ & 1570 & & \\
\hline Total & 7211 & & 3542 & & 3119 & & 2872 \\
\hline
\end{tabular}

* Age categories between the countries differ because the usual local age categorisations were used.

$<2$ years) were measured with a non-stretchable flexible tape. Information about lean and fat mass was also collected using bioelectrical impedance (only Malaysia) and DXA in a subsample of urban participants in all the countries. Blood pressure was measured only in Malaysian children. An overview of the study parameters and methodology for each country is presented in Table 4.

\section{Data collection and management}

Each of the countries used mobile field teams to visit the identified regions. Each team consisted of enumerators with knowledge of nutrition and/or biomedical analysis. Enumerators in these teams were centrally trained on the objectives of the study, anthropometric measurements, clinical examinations, techniques of interviews, sequence of questionnaires and its variables, sampling procedures, field management and, at a later stage, data entry, checking and cleaning. Psychologists specialised in child development (Thailand) or well-trained enumerators (other countries) administered the mental and cognitive development tests.

Data entry was done using specially developed programmes (Indonesia and Thailand), Epi Info (Malaysia) and EpiData 3.1 (Vietnam). Furthermore, Excel files with a fixed format were used by all countries for the final overview of data. At least $10 \%$ of data had a double entry made by different persons to check the accuracy of data entry. Excel files, statistical data files and other information related to the study were uploaded on a specially secured SEANUTS website on a regular basis where all data were accessible for the project team and per country for the specific research teams.

Monitoring took place online by checking the upload of data, as well as by regular field visits made by one of the project team members. Country-by-country progress reports were prepared when particular regions in the countries were finalised. Regional meetings with research team delegations and project team members were organised every $6-8$ months.
In cooperation with the institutes involved in the study, data obtained from this survey can be made accessible for governments and teaching and scientific projects on request (A. S.).

\section{Materials and methods}

\section{Anthropometry}

Body weight was measured using digital weighing scales to the nearest $0.1 \mathrm{~kg}$, and height and sitting height were measured using a stadiometer or microtoise to the nearest $0 \cdot 1 \mathrm{~cm}$. For height measurements, children were measured standing straight and bare footed, with their heels (together), shoulder, back and buttocks touching the wall. In children aged $<2$ years, length to the nearest $0 \cdot 1 \mathrm{~cm}$ was measured using a wooden measuring board. Measured weight and height were used to calculated the appropriate WHO $z$-scores for age ${ }^{(9,10)}$.

\section{Body composition}

BMI was calculated as weight $(\mathrm{kg}) /$ height squared $\left(\mathrm{m}^{2}\right)$. From sitting height, relative leg length was calculated as $1-$ (sitting height/height). From height and the skeletal widths (as measured to the nearest $0 \cdot 1 \mathrm{~cm}$ with calipers) of wrist and knee, the slenderness index was calculated as height/(sum of wrist and knee widths). A higher index indicates a more slender body build. Relative leg lengths and slenderness are known to have an impact on the interpretation of the $\mathrm{BMI}^{(11,12)}$. In Malaysia, the body composition of children was also measured using bioelectrical impedance equipment. Validation was carried out in a subsample of participants against a total-body DXA scan. In the other countries, only full-body DXA scans were taken in subpopulations to study body composition.

Mid-upper arm circumference and waist circumference were measured using a non-stretchable flexible tape to the nearest $0 \cdot 1 \mathrm{~cm}$. Mid-upper arm circumference is a parameter used for the identification of undernutrition among children aged $<5$ years. Waist circumference is an identifier for

Table 3. Number of participants in each country by sex and area of residence

\begin{tabular}{llcccrr}
\hline Sex & Area & Indonesia & Malaysia & Thailand & Vietnam & Total \\
\hline Girls & Urban & 1666 & 1050 & 497 & 715 & 3928 \\
& Rural & 1829 & 730 & 1054 & 715 & 4328 \\
Boys & Urban & 1787 & 1052 & 505 & 720 & 4064 \\
& Rural & 1929 & 710 & 1063 & 722 & 4424 \\
Total & 7211 & 3542 & 3119 & 2872 & 16744 \\
\hline
\end{tabular}


Table 4. Overview of South East Asian Nutrition Survey (SEANUTS) measurements, materials and methods for Indonesia, Malaysia, Thailand and Vietnam

\begin{tabular}{|c|c|c|c|c|}
\hline & Indonesia & Malaysia & Thailand & Vietnam \\
\hline Study design & Cross-sectional & Cross-sectional & Cross-sectional & Cross-sectional \\
\hline Data collection & January-December 2011 & May 2010-October 2011 & January-August 2011 & March-May 2011 \\
\hline $\begin{array}{l}\text { Age of the } \\
\text { subjects (years) }\end{array}$ & $0.5-12$ & $0.5-12$ & $0.5-12$ & $0.5-11$ \\
\hline \multicolumn{5}{|l|}{ Sample size/grouping ( $n$ ) } \\
\hline & $0.5-1$ years: 2392 & $0.5-0.9$ years: 68 & $0.5-0.9$ years: 142 & $0.5-5.9$ years: 952 \\
\hline & $2-5$ years: 2413 & $1-3$ years: 538 & $1-2$ years: 547 & $6-11.9$ years: 1920 \\
\hline & $6-12$ years: 2406 & $4-6$ years: 967 & $3-5$ years: 860 & Total: 2872 \\
\hline & Total: 7211 & $7-12$ years: 1969 & $6-12$ years: 1570 & \\
\hline & & Total: 3542 & Total: 3119 & \\
\hline Sampling method & $\begin{array}{l}\text { Population-based, multistage cluster } \\
\text { sampling: districts ( } 10 \%) \text { and villages } \\
\text { (four per district) }\end{array}$ & $\begin{array}{l}\text { School-, kindergarten- and nursery-based } \\
\text { stratified random sampling }\end{array}$ & $\begin{array}{l}\text { Population-based, multistage cluster } \\
\text { sampling: regions (four plus Bangkok) and } \\
\text { provinces }\end{array}$ & $\begin{array}{l}\text { School-, kindergarten- and nursery- } \\
\text { based multistage cluster sampling: } \\
\text { Northern, Central and Southern } \\
\text { regions }\end{array}$ \\
\hline Data entry & $\begin{array}{l}\text { In-house developed data entry program } \\
\text { based on FoxproDouble entry }(10 \%) \\
\text { to ensure the accuracy of data entry }\end{array}$ & $\begin{array}{l}\text { Epi InfoDouble entry (10\%) to ensure the } \\
\text { accuracy of data entry }\end{array}$ & $\begin{array}{l}\text { In-house developed program based on } \\
\text { Microsoft Access 2007Double entry (10\%) } \\
\text { to ensure the accuracy of data entry }\end{array}$ & $\begin{array}{l}\text { EpiData 3.1Double entry }(20 \%) \text { to } \\
\text { ensure the accuracy of data entry }\end{array}$ \\
\hline $\begin{array}{l}\text { Socio-economic/demo- } \\
\text { graphic information }\end{array}$ & SEQ & SEQ & SEQ & $\begin{array}{l}\text { SEQ prepared by the National Institute } \\
\text { of Nutrition }\end{array}$ \\
\hline $\begin{array}{l}\text { Health status of the } \\
\text { child }\end{array}$ & $\mathrm{CHSQ}$ & CHSQ & CHSQ & No \\
\hline Dietary intake & $\begin{array}{l}\text { Age-specific FFQ } 24 \mathrm{~h} \text { food recall ( } n \\
3600) \\
\text { BCFQ }\end{array}$ & Age-specific FFQBCFQ & Age-specific FFQ $24 \mathrm{~h}$ food recall $B C F Q$ & $24 \mathrm{~h}$ food recall for $1-3 \mathrm{~d}$ \\
\hline Nutrient calculation & $\begin{array}{l}\text { Software: Nutrisoft program developed by } \\
\text { the Indonesian Nutrition Research Cen- } \\
\text { ter using Indonesian food composition } \\
\text { table }\end{array}$ & $\begin{array}{l}\text { Software: FFQcal. Nutrient Composition of } \\
\text { Malaysian Foods; Singapore Food } \\
\text { Composition (2001); ASEAN Food } \\
\text { Composition Table (2000); USDA National } \\
\text { Nutrient Database for Standard Reference } \\
\text { Release } 23 \text { (2010); food labels }\end{array}$ & $\begin{array}{l}\text { Software: INMUCAL, Institute of Nutrition, } \\
\text { Mahidol University }\end{array}$ & $\begin{array}{l}\text { Software: Nurisoft program developed } \\
\text { by the National Institute of Nutrition, } \\
\text { using the Vietnamese food compo- } \\
\text { sition table }\end{array}$ \\
\hline Food habits & No & CFHQ & CFHQ & CFHQ \\
\hline Weight & SECA digital weighing scale; Seca & SECA 803, digital weighing scale; Seca & SECA 822, digital weighing scale; Seca & $\begin{array}{l}\text { A\&D UC } 321 \text { digital weighing scale } \\
\text { (A\&D Weighing) }\end{array}$ \\
\hline Height & Harpenden microtoise (Holtain, UK) & SECA 213, portable stadiometer; Seca & Microtoise; Stanley-Mabo & $\begin{array}{l}\geq 2 \text { years: wall-mounted stadiometer; } \\
\text { Holtain }<2 \text { years: wooden measuring } \\
\text { board for length; Helen Keller Organ- } \\
\text { ization }\end{array}$ \\
\hline Sitting height & Stretch stature method & SECA 206 Bodymeter; Seca & Microtoise; Stanley-Mabo & Wall-mounted stadiometer; Holtain \\
\hline Upper arm length & No & Lufkin W606PM tape (Cromwell, UK) & No & No \\
\hline Breadths & $\begin{array}{l}\text { Breadth caliper, Harpenden Elbow, } \\
\text { wrist and knee }\end{array}$ & $\begin{array}{l}\text { Breadth caliper, Harpenden Elbow, wrist and } \\
\text { knee }\end{array}$ & $\begin{array}{l}\text { Breadth caliper; HoltainElbow, wrist and } \\
\text { knee }\end{array}$ & $\begin{array}{l}\text { Breadth caliper, Harpenden Elbow, } \\
\text { wrist and knee }\end{array}$ \\
\hline Circumferences & $\begin{array}{l}\text { Flexible tape, non-stretchable } \\
\text { Mid-upper arm }\end{array}$ & $\begin{array}{l}\text { Lufkin W606PM tape Mid-upper arm, waist, } \\
\text { head ( } \leq 4 \text { years) and hip }\end{array}$ & $\begin{array}{l}\text { Flexible tape, SECA 201; Seca Mid-upper } \\
\text { arm and waist }\end{array}$ & Flexible tape Mid-upper arm \\
\hline Skinfolds & $\begin{array}{l}\text { Skinfold caliper; HoltainTricipital, } \\
\text { subscapular, bicipital and suprailiac }\end{array}$ & $\begin{array}{l}\text { Skinfold caliper, Harpendentricipital and sub- } \\
\text { scapular }\end{array}$ & $\begin{array}{l}\text { Skinfold caliper; Holtain Tricipital, subscapu- } \\
\text { lar, bicipital and suprailiac }\end{array}$ & $\begin{array}{l}\text { Skinfold caliper; Holtain Tricipital, } \\
\text { subscapular, bicipital and suprailiac }\end{array}$ \\
\hline Handgrip & No & Yes & No & No \\
\hline Body composition & $\begin{array}{l}\text { DXA, Lunar Prodigy Pro (GE Healthcare), } \\
\text { Program Encore 2008, version 12.30; } \\
\text { General ElectricFull-body scan, } n 100\end{array}$ & $\begin{array}{l}\text { DXA; HologicFull-body scan, } n 200 \\
\text { Bioelectrical impedance: Bodystat } \\
1500 \text { MDD, } n 3505\end{array}$ & $\begin{array}{l}\text { DXA, Lunar Prodigy Pro (GE Healthcare), } \\
\text { Program Encore 2008, version } 12.30 \\
\text { Full-body scan, } n 184\end{array}$ & $\begin{array}{l}\text { DXA, Explorer (S/N 90530); HologicFull- } \\
\text { body scan, } n 295\end{array}$ \\
\hline Bone density/quality & $\begin{array}{l}\text { Quantitative ultrasound, Omnisense, } \\
\text { 7000P; Sunlight Medical }\end{array}$ & $\begin{array}{l}\text { Quantitative ultrasound, Omnisense } \\
\text { 8000P Mobile; Sunlight Medical }\end{array}$ & $\begin{array}{l}\text { Quantitative ultrasound, Omnisense } 8000 \mathrm{P} \\
\text { Mobile; Sunlight Medical }\end{array}$ & $\begin{array}{l}\text { Quantitative ultrasound, Omnisense } \\
\text { 8000P Mobile; Sunlight Medical }\end{array}$ \\
\hline Physical activity & $\begin{array}{l}\text { PAQ, age specific (adopted from Malay- } \\
\text { sia)Pedometer ( } n \text { 2400), Digi-walker } \\
\text { CW-700; Yamax }\end{array}$ & $\begin{array}{l}\text { PAQ, age specific Pedometer } \\
\text { (n 819), Digi-walker CW-700; Yamax }\end{array}$ & $\begin{array}{l}\text { PAQ1 (1-3 years), PAQ2 (4-6 years) } \\
\text { (adopted from Malaysia) and PAQ3 }(7-12 \\
\text { years), validated in ThailandPedometer } \\
\text { ( } n \text { 897), Omron HJ 203; Omron }\end{array}$ & $\begin{array}{l}\text { PAQ, age specific (adopted from Malay- } \\
\text { sia)Pedometer ( } n \text { 805), Tanita PD- } \\
\text { 641; Tanita }\end{array}$ \\
\hline
\end{tabular}




\section{N British Journal of Nutrition}

Table 4. Continued

\begin{tabular}{|c|c|c|c|c|}
\hline & Indonesia & Malaysia & Thailand & Vietnam \\
\hline \multicolumn{5}{|l|}{ Blood } \\
\hline Age & $\begin{array}{r}<2 \text { years, finger prick for } \mathrm{Hb}(n 750) \\
>2 \text { years, venous blood }(n 1500)\end{array}$ & $>3$ years, $n 872$ & $\begin{array}{c}<3 \text { years, finger prick for } \mathrm{Hb}(n \text { 132) } \\
\geq 3 \text { years, venous blood }(n 495)\end{array}$ & $\begin{array}{l}\leq 5.9 \text { years, finger prick for } \mathrm{Hb} \\
\quad(n \text { 192) } \geq 6 \text { years, venous blood } \\
(n \text { 386) }\end{array}$ \\
\hline Full blood count & Yes & Yes & Yes & Yes \\
\hline $\begin{array}{l}\text { Total Fe-binding } \\
\text { capacity }\end{array}$ & No & Serum transferrin $\times 22.4$ & No & No \\
\hline $\mathrm{Hb}$ & $\begin{array}{l}\text { HemoCue Hb } 201 \text { (HemoCue } \\
\text { Diagnostics B.V.; <2 years) } \\
\text { Spectrophotometry, ADVIA 2120; } \\
\text { Siemens }\end{array}$ & $\begin{array}{l}\text { Spectrophotometry, Sysmex XE5000; } \\
\text { Sysmex }\end{array}$ & $\begin{array}{l}\text { HemoCue Hb } 201 \text { (HemoCue Diagnostics } \\
\text { B.V.; <3 years) Colorimetric method } \\
\text { (automated analyser, Minray BC 5180; } \\
\text { Minray) }\end{array}$ & $\begin{array}{l}\text { HemoCue } 201 \text { (HemoCue } \\
\text { Diagnostics B.V.; } \leq 5.9 \text { years) } \\
\text { Spectrophotometry, ADVIA 2120; } \\
\text { Siemens ( } \geq 6 \text { years) }\end{array}$ \\
\hline Serum ferritin & $\begin{array}{l}\text { Immunochemiluminescence ECLIA, } \\
\text { Roche Cobas e 601; Roche Diagnos- } \\
\text { tics }\end{array}$ & $\begin{array}{l}\text { Immunochemiluminescence, ADVIA } \\
\text { Centaur; Siemens }\end{array}$ & $\begin{array}{l}\text { Electrochemiluminescence immunoassay, } \\
\text { Cobas }^{\circledR} ; \text { Roche Diagnostics, GmbH }\end{array}$ & $\begin{array}{l}\text { Roche, Cobas c 601, ELISA; Ramco } \\
\text { Laboratories, Inc.Plate reader: } \\
\text { Biotek ELx808 (BioTek) }\end{array}$ \\
\hline $\begin{array}{l}\text { Soluble transferrin } \\
\text { receptors }\end{array}$ & No & No & $\begin{array}{l}\text { Enzyme Immunoassay; Ramco } \\
\text { Laboratories, Inc. }\end{array}$ & $\begin{array}{l}\text { Roche, Cobas c 501, ELISA; Ramco } \\
\text { Laboratories, Inc.Plate reader: } \\
\text { Biotek ELx808 (BioTek) }\end{array}$ \\
\hline Transferrin & No & Immunoturbidimetry, ADVIA 2400; Siemens & & \\
\hline C-reactive protein & $\begin{array}{l}\text { Immunoturbidimetry, Cobas Integra; } \\
\text { Roche }\end{array}$ & Immunoturbidimetry, ADVIA 2400; Siemens & $\begin{array}{l}\text { Immunonephelometry, CardioPhase }{ }^{\circledR ;} \\
\text { Siemens }\end{array}$ & $\begin{array}{l}\text { Roche, Cobas c 501, ELISA; } \\
\text { Immunology Consultants Laboratory, } \\
\text { Inc.Plate reader: Biotek ELx808 }\end{array}$ \\
\hline$\alpha-1$ Glycoprotein & $\begin{array}{l}\text { Immunoturbidimetry, Tina-quant } \alpha-1 \text { Acid } \\
\text { Glycoprotein Gen.2, Cobas Integra; } \\
\text { Roche }\end{array}$ & Immunoturbidimetry; Integra & ELISA; GenWay Biotech, Inc. & $\begin{array}{l}\text { ELISA; Immunology Consultants } \\
\text { Laboratory, Inc. Plate reader: } \\
\text { Biotek ELx808 (BioTek) }\end{array}$ \\
\hline Serum retinol & $\begin{array}{l}\text { HPLC-UV detector, Agilent, 1200; Agilent } \\
\text { Technologies (all-trans-retinol) }\end{array}$ & $\begin{array}{l}\text { HPLC, Agilent 1100; Agilent Technologies } \\
\text { (all-trans-retinol) }\end{array}$ & $\begin{array}{l}\text { HPLC/Photodiode Array Detector; Waters } \\
\text { Corporation (all-trans-retinol) }\end{array}$ & $\begin{array}{l}\text { HPLC, LC-10 ADVP; Shimadzu } \\
\text { (all-trans-retinol) }\end{array}$ \\
\hline Serum $25(\mathrm{OH}) \mathrm{D}$ & $\begin{array}{l}\text { ELISA, IDS 25-Hydroxy Vitamin } \\
\text { D; Immunodiagnostic Systems }\left(D_{3} \text { and }\right. \\
\left.D_{2} \text { metabolites }\right)\end{array}$ & $\begin{array}{l}\text { CLIA, LIAISON }{ }^{\circledR} 25 \text { OH Total Vitamin D; } \\
\text { Diasorin, Inc. ( } \mathrm{D}_{3} \text { and } \mathrm{D}_{2} \text { metabolites) }\end{array}$ & $\begin{array}{l}\text { CLIA, LIAISON }{ }^{\circledR} 25 \mathrm{OH} \text { Total Vitamin D; } \\
\text { Diasorin, Inc. ( } \mathrm{D}_{3} \text { and } \mathrm{D}_{2} \text { metabolites) }\end{array}$ & $\begin{array}{l}\text { HPLC, ECLIA, Alliance; Waters. } \\
\text { Cobas e } 601 ; \text { Roche Diagnostics } \\
\left.\text { ( } D_{3} \text { metabolite only }\right)\end{array}$ \\
\hline $\begin{array}{l}\text { DHA (in erythro- } \\
\text { cytes) }\end{array}$ & $\begin{array}{l}\text { Capillary } \\
\text { tion } \\
\text { ti617) }^{(10 \% \text { flame of blood samples })}\end{array}$ & $\begin{array}{l}\text { Capillary GC/flame ionisation detection; } \\
\text { Gribbles Laboratories }\end{array}$ & Capillary GC/flame ionisation detection & Capillary GC/flame ionisation detection \\
\hline Lipid profile & No & No & $\begin{array}{l}\text { TC, LDL-cholesterol and HDL-cholesterol: } \\
\text { enzymatic method, Biosystems; Biosystem } \\
\text { SATAG: enzymatic method; Beckman } \\
\text { Coulter }\end{array}$ & No \\
\hline Blood pressure & No & $\begin{array}{l}\text { Digital sphygmomanometer, HEM-907; } \\
\text { Omron }\end{array}$ & No & No \\
\hline Mental development & $\begin{array}{l}\text { Bailey-3 (6-42 months) DENVER II } \\
\text { (6-66 months) }\end{array}$ & $\begin{array}{l}\text { Ages and stages questionnaire (ASQ-3) } \\
\text { (6-47 months) }\end{array}$ & DENVER II (0.5-6 years) & ASQ-3 \\
\hline Cognition & $\begin{array}{l}\text { WISC-3: WPPSI ( } 3 \text { years ( } 10 \text { months, } \\
16 \text { d) }-5.9 \text { years); WISC- } 3 \quad(6 \cdot 0-12 \\
\text { years) Raven's Standard Progressive } \\
\text { Matrices test ( } 5 \cdot 5-12 \text { years) }\end{array}$ & $\begin{array}{l}\text { Raven's Coloured Progressive Matrices test } \\
\text { (4-11 years) Raven's Standard Progress- } \\
\text { ive Matrices test ( } \geq 11 \text { years) }\end{array}$ & $\begin{array}{l}\text { Test of Nonverbal Intelligence, Third Edition } \\
\text { (TONI-3) }(6-12 \text { years) }\end{array}$ & Raven's Progressive Matrices test \\
\hline lodine in urine & $\begin{array}{l}\text { Spectrophotometry; KC4 Program } \\
\text { (school children) }\end{array}$ & No & No & No \\
\hline
\end{tabular}

SEQ, socio-economic questionnaire; CHSQ, Child Health Status Questionnaire; BCFQ, Breast-feeding and Complementary Feeding Questionnaire; CFHQ, Child Food Habit Questionnaire; DXA, dual-energy X-ray absorptiometry; PAQ, Physical Activity Questionnaire; ECLIA, electrochemiluminescent immunoassay; 25(OH)D, 25-hydroxyvitamin D; IDS, immunoactivity detection system; TC, total cholesterol; ASQ-3, Ages and Stages Questionnaire-3;
WISC-3, Wechsler Intelligence Scale for Children-3; WPPSI, Wechsler Preschool and Primary Scale of Intelligence; TONI-3, Test of Nonverbal Intelligence, Third Edition. 
overnutrition and body fat distribution. Head circumference was measured in only Malaysia for participants aged $<2$ years using a non-stretchable flexible tape $(0 \cdot 1 \mathrm{~cm}$ accurate). Tricipital and subscapular skinfolds, and in addition to bicipital and suprailiac ones in Vietnam, Thailand and Indonesia, were measured using skinfold calipers to the nearest $0.1 \mathrm{~mm}$. Data will be used to predict body fat (\%) after validation of the published prediction equations against DXA measurements in each country. BMI, circumference and skinfold measurements were also calculated as WHO $z$-scores for age ${ }^{(9,10)}$.

\section{Bone}

Bone quality was measured using a quantitative ultrasound technique in the tibia and/or the radius following the instructions of the manufacturer. In a subsample of children, a full-body scan (bone mineral density and fat mass - lean mass) using DXA was also taken for the validation of the quantitative ultrasound method.

\section{Physical activity}

Four sets of age-specific physical activity questionnaires were used to assess the physical activity pattern of children. These questionnaires, modifications of existing questionnaires for the age groups 2-3, 4-6, 7-9 and 10-12 years, were pre-tested and validated with an accelerometer during the preparation phase. For a more objective physical activity measurement, a subsample of participants wore pedometers for seven consecutive days to count the number of steps. During the handout of pedometers, parents and children were instructed on how to use them. The display of pedometers was taped off to prevent a read-out by parents and children during the study period. Directly after the measurement period, pedometers were collected, and compliance was checked in an interview with the children and/or parents.

\section{Sociodemographic background and general health status}

A set of questionnaires on sociodemographic data, including the level of parental education and income, were filled in by the parent(s) of the subjects. The health status questionnaire included question related to infections and defaecation and other questions related to child health. All questionnaires were interviewer- or self (parents)-administered. For children aged 6 months -9 years, the parents or carers were interviewed, while children aged 10 years and above were interviewed with assistance from their parents, when necessary.

\section{Dietary intake and food habits}

Dietary intake was assessed using a semi-quantitative FFQ or $24 \mathrm{~h}$ recall. A total of five sets of age-specific FFQ (6-23 months, 2-6 years and 7-12 years; Thailand used two sets: for children aged $<3$ and $>3$ years) were developed, validated in the preparation phase by comparing with a $3 \mathrm{~d}$ recall method and adapted for country-specific food items.
Based on this comparison, the mean intake of macronutrients was comparable between the FFQ and the recall method, although the reported intakes measured using the FFQ tended to be higher. The repeatability of FFQ outcome was checked and approved in the preparation phase by providing the FFQ again 1 week after the initial completion. To facilitate accurate reporting, written instructions on how to fill in the FFQ were provided and photographs of food portion sizes were given in a separate booklet. For younger children (6 months-9 years), the FFQ was completed by their mothers or other carers. Older children aged 10 years and above completed the FFQ by themselves. The FFQ was provided to the parents or carers before the day of physical measurements. On the day of the measurements, the FFQ was collected and checked for completeness and, where necessary, the information was verified with the parents or carers. FFQ data or recall data were converted into nutrient intakes using computer software programs containing the nutrient compositions of local and/or regional products.

A total of two sets of child food habit questionnaires were used to obtain descriptive data on dietary intakes of children aged 2 years and above (one set administered to parents of children aged 2-9 years and another set for children aged 10-12 years). For children aged 6-23 months, a breastfeeding and complementary feeding questionnaire was answered by their mothers.

\section{Mental development and cognitive function}

Mental development of children from 6 to 66 months was assessed using appropriate and available tests and questionnaires. Cognitive function was assessed in children from 4 or 6 years onwards, depending on the available tests in each country (Table 4). The choices of questionnaires and tests were determined by the available expertise in the countries.

\section{Biochemical analysis and blood pressure}

Venous blood was collected from a 20 to $30 \%$ random subsample (based on date of entry, sex and age) of total children from the age of 2 years onwards in Indonesia, from that of 3 years onwards in Thailand and Malaysia, and from that of 6 years onwards in Vietnam. The samples were stored on ice and as soon as possible sent to accredited laboratories for immediate processing. Erythrocyte preparation for DHA analysis was done on-site. Blood samples were immediately analysed or prepared and frozen until analysis. The choice of the analytical method was based on the instrumentation and expertise available in the laboratory in each country. For vitamin D, Diasorin Liaison total vitamin D assay, Roche electrochemiluminescent immunoassay (ECLIA) and immunoactivity detection system (IDS) 25-Hydroxy Vitamin D were used. These methods have known differences in outcomes and results have to be corrected accordingly for inter-country comparisons $^{(13,14)}$

Excess plasma was frozen for future analysis of micronutrient deficiencies depending on dietary recall results. 


\section{Statistics}

This section describes how the participants were recruited in each of the four countries, which weight factor calculation was carried out, how the data were collected, which statistical methods were used and how the power analysis was done.

Sampling method and recruitment. In all the countries, a cross-sectional study design was applied, in both urban and rural areas. Indonesia, Thailand and Vietnam used a multistage cluster sampling approach, while Malaysia used a stratified random sampling approach. Urban and rural areas were taken as strata and the various (sub)regions per country as clustering units. The principal clustering unit was the demographic unit with the highest number of inhabitants. Various demographic clustering units per country were found to be of importance within the present survey: Indonesia - country to districts (forty-eight of 440 districts, 150 subjects per district) and subsequently clusters (villages, four per district, rural and urban); Thailand - country to region (Central, Northern, Northeastern and Southern regions and Bangkok, with two provinces in the Northeastern region and one province in the Central, Northern and Southern regions), and each region in Thailand contained municipalities (urban) and non-municipalities (rural) and was finally divided into district and subdistricts; Vietnam: country to region (Northern, Central and Southern regions, with each region containing urban and rural communities) with one province and finally one district per province; Malaysia: country to region (Northern, Central, Southern, East Coast, Sabah and Sarawak regions, with each region containing urban and rural locations), subsequently to educational centre level (six schools, six kindergartens and four nurseries per location) and finally to the age group level (four groups with 360 children in each urban location and 240 children in each rural location).

Both Indonesia and Thailand used population-based sampling approaches, whereas Malaysia and Vietnam sampled on the school, kindergarten and nurseries levels.

Weight factor calculation was done on the basis of the number of boys and girls per age group defined per regional unit as representative to the total number of children in the next demographic unit and to finally the national level. This was done according to the demographic information per country available during the last national census, in all cases that of 2010 (Indonesia: Statistics Indonesia of The Republic Indonesia, Jakarta; Thailand: National Statistical Office, Bangkok; Vietnam: General Statistics Office of Vietnam, Hanoi; and Malaysia: Department of Statistics, Putrajaya, Kuala Lumpur). To correct for the increase in the number of children per age group in 2011, when the sampling in the present study was performed, the national growth rate, potentially adjusted for the age range, was used as provided in the same national census in 2010 for all the countries.

Data collection was done by the local teams per country and stored in Excel using preformatted tables. At regular intervals, these data tables were cleaned and checked for obvious outliers, typing errors and missing data using specific tools within STATA version 12 (StataCorp), SPSS version 18 (IBM Corporation) and Excel (Microsoft Corporation). Accordingly, missing values have been coded and are not included in the final evaluation. Outliers were checked for potential effects on the various population-based results and discussed with the representatives per country. The various descriptive values are all weighed for population size/distribution, and mean values have been tested for differences after correction for age and/or area of residence where appropriate.

Statistical methods. Data from the surveys in each of the four countries are presented as descriptive values and as standardised scores where appropriate. Correlation analysis was carried between the parameters of interest. The interrelationship between parameters was studied using stepwise multiple regression $^{(15)}$, accounting for confounding. Depending on the distribution pattern of the dependent variable, a standard, logistic or Poisson regression was applied, taking confounding factors into account. The analyses were done per boys or girls and/or per age-year, age-group or any other relevant combination. Moreover, to analyse potential differences between the selected groups, dummy parameters were introduced to account for differences between the groups or differences changing over time (age-years). Age adjustment of data was done using standardisation techniques if appropriate for analysis. Throughout the study, a $P$ value of 0.05 was considered to be statistically significant applying two-sided testing.

Power analysis. The number of participants to be included in each of the four surveys was based on the potential occurrence of anaemia (18-25\%) and/or vitamin A deficiency (19-22\%) and/or underweight (9-18.4\%), depending on the country's interest and likely nutritional problems.

The formula used was as follows:

$$
N=Z^{2} \times p(1-p) \times \mathrm{DEFF} /(\text { tolerable error })^{2},
$$

in which $N$ is the number of participants per age class, $Z$ is the confidence level (1.96), $p$ is the prevalence (\%) of the particular condition, DEFF is the estimated design effect (ratio of actual variance within the sampling method used to that of the simple random sampling method), and tolerable error is the level of specificity (usually ranging between 1.8 for underweight and $4 \cdot 0$ for anaemia).

Since per country different conditions were applied, the number of participants per country was based on the sensitivity of finding the highest prevalence.

\section{Discussion}

The primary aim of the SEANUTS, obtaining an updated overview of the nutritional and health status of older infants and children, was achieved. Valuable information is and will become available for public health policies and nutrition research and development. The secondary aim, comparing the results for the four participating countries, was achieved with regard to especially the anthropometric, physical activity and nutritional status parameters.

In the organisation of the SEANUTS, regular meetings with all the principal investigators and their researchers and the project team were of great help. Besides the exchange of 
information, these meetings facilitated the strengthening of relationships between the research institutes. This might lead to closer collaborations in the long run, which should be beneficial for the regions. The regular field visits made by the project team, especially in the early stages of the project, also contributed to the success of the project. In retrospect, we must conclude that extra attention should have been paid to comparability of the study protocols to maximise the project outcomes. For example, it is difficult to make straightforward comparisons between the countries with regard to the parameters of cognitive performance and mental development due to the differences in the methodology used.

Analytical methods could not be fully aligned within the time frame of the project. The major reason for this was that it would take quite some time before the laboratories involved were on the same and appropriate quality level for all the methods used. At that time, it seemed wise to choose for experience on existing methodology. Another limitation in comparing data is the absence of a ring test for the most important biochemical parameters. In practice, this proved to be very difficult since transport of blood samples was not allowed by some countries and transport on dry ice of ring test samples failed (samples were thawed at arrival).

In all the four countries, the participants were representative for the target population. However, sampling methods differed slightly. The major difference might be between population-based sampling and school-kindergarten-nurserybased sampling. It is to be expected that a population-based strategy, including also the very low-socio-economic status families that do not attend nurseries, kindergartens or even schools, will affect the results of most of the parameters studied.

Data collection was carried out successfully and the secured website for uploading information proved to be extremely useful. Despite this, the study took about 6 months longer than initially foreseen. The main reason for this was the underestimation of the time needed for data cleaning, questionnaire evaluation, statistical evaluation and finally the preparation of the reports.

In conclusion, the SEANUTS provides data that will be very relevant for assessing the effect of current health and nutrition programmes or that can be used to start such programmes. Insights into growth parameters in combination with nutritional status and dietary habits provide relevant information for food companies to develop new products or to fine-tune the existing ones. In the end, the SEANUTS will benefit the target population in the countries.

\section{Acknowledgements}

The authors are indebted to the research teams of each of the countries involved, as well as to the parents/carers, infants and children for their willingness to participate in the study.

FrieslandCampina provided funding for the SEANUTS, but it was not involved in the recruitment of participants, cognitive performance testing and the final set of results.

A. S. was the project leader and participated in the writing of the study protocols as well as evaluation of the study results; waist circumference supervised the statistical analysis; E. G. H. M. v. d. H. was involved in the choosing of the biochemical methods used; C. v. B., J. H. and P. D. identified the research institutes and were involved in the writing of the study protocols; P. D., I. K. and P. P. were involved in data management, study monitoring and data evaluation; S., P. B. K., N. R. and B. K. L. N. were involved in the writing and execution of the study protocols and were the principal investigators. All authors took part in the writing of this manuscript.

Conflicts of interest: The results of the study will be used by FrieslandCampina, but it had no influence on the outcome of the study. None of the other authors or the research institutes has any conflicts of interest.

This paper was published as part of a supplement to the British Journal of Nutrition, the publication of which was supported by an unrestricted educational grant from Royal FrieslandCampina. The papers included in this supplement were invited by the Guest Editor and have undergone the standard journal formal review process. They may be cited. The Guest Editor appointed to this supplement is Dr Panam Parikh. The Guest Editor declares no conflict of interest.

\section{References}

1. Cordain L, Eaton SB, Sebastian A, et al. (2005) Origins and evolution of the Western diet: health implications for the 21st century. Am J Clin Nutr 81, 341-354.

2. Christian P \& Stewart CP (2010) Maternal micronutrient deficiency, fetal development, and the risk of chronic disease. J Nutr 140, 437-445.

3. Eilander A, Gera T, Sachdev HS, et al. (2010) Multiple micronutrient supplementation for improving cognitive performance in children: systematic review of randomized controlled trials. Am J Clin Nutr 91, 115-130.

4. Hollis B (2005) Circulating 25-hydroxyvitamin D levels indicative of vitamin D sufficiency: implication for establishing a new effective dietary intake recommendation for vitamin D. J Nutr 135, 317-322.

5. U.S. Department of Health and Human Services, Centers for Disease Control and Prevention \& National Center for Health Statistics (2012). National Health and Nutrition Examination Survey (2007-2008). http://www.cdc.gov/nhanes

6. Vincente-Rodriguez G, Libersa Chr, Mesana MI, et al. (2007) Healthy Lifestyle by Nutrition in Adolescence (HELENA). A new EU funded project. Thérapie 62, 259-270.

7. Briefel RR, Kalb LM, Condon E, et al. (2010) The Feeding Infants and Toddlers Study 2008: study design and methods. $J$ Am Diet Assoc 110, s16-s26.

8. Thurhnam Dl, McCabe LD, Haldar S, et al. (2010) Adjusting plasma ferritin concentrations to remove the effects of subclinical inflammation in the assessment of iron deficiency: a meta-analysis. Am J Clin Nutr 92, 546-555.

9. Norgan NG (1994) Population differences in body composition in relation to BMI. Eur J Clin Nutr 48, s10-s27.

10. Deurenberg P, Deurenberg-Yap M, Wang J, et al. (1999) The impact of body build on the relationship between body mass index and body fat percent. Int $J$ Obesity $\mathbf{2 3}$ 537-542.

11. WHO (2006) The WHO child growth standards. http://www. who.int/childgrowth/standard/bmi_for_age/en/index.html

12. WHO (2007) Growth reference data for 5-19years. http://www who.int/growthref/who2007_bmi_for_age/en/index.html 
13. Leino A, Turpeinen U \& Koskinen P (2008) Automated measurement of $25-\mathrm{OH}$ vitamin $\mathrm{D}_{3}$ on the Roche Modular E170 analyzer. Clin Chem 54, 2059-2062.

14. Connell AB, Jenkins N, Black M, et al. (2011) Overreporting of vitamin $\mathrm{D}$ deficiency with the Roche Elecsys vitamin $\mathrm{D}_{3}$ (25-OH) method. Pathology 43, 368-371.

15. Kleinbaum DG, Kupper LL, Muller KE, et al. (1998) Applied Regression Analysis and Other Multivariable Methods, 3rd ed. Pacific Grove, CA: Duxbury Press.
16. Muskiet FAJ, Van Doormaal JJ, Martini IA, et al. (1983) Capillary gas chromatographic profiling of total longchain fatty acids and cholesterol in biological materials. I Chromatogr B 278, 231-244.

17. Van der Steege G, Muskiet FAJ, Martini IA, et al. (1987) Simultaneous quantification of total medium- and long-chain fatty acids in human milk by capillary gas chromatography with split injection. $J$ Chromatogr $B \mathbf{4 1 5}$, $1-11$. 